\title{
A Zirconium Metal-Organic Framework with SOC Topological Net for Catalytic Peptide Bond Hydrolysis
}

Sujing Wang ( $\nabla$ sjwang4@ustc.edu.cn )

IMAP ENS Paris https://orcid.org/0000-0003-0942-2907

Hong Giang T Ly

KU Leuven

\section{Mohammad Wahiduzzaman}

Institut Charles Gerhardt Montpellier, Université Montpellier https://orcid.org/0000-0003-2025-4115

lurii Dovgaliuk

IMAP ENS Paris https://orcid.org/0000-0003-1997-4748

Antoine Tissot

IMAP ENS Paris

Guillaume Maurin

Institut Charles Gerhardt, CNRS ENSCM, Université Montpellier

Tatjana Parac-Vogt

KU Leuven https://orcid.org/0000-0002-6188-3957

Christian Serre

IMAP ENS Paris

\section{Article}

Keywords: Nanozymes, Selective Fragmentation of Proteins, Modern Proteomics, Reusable Artificial Catalysts

Posted Date: June 16th, 2021

DOl: https://doi.org/10.21203/rs.3.rs-470618/v2

License: (c) (i) This work is licensed under a Creative Commons Attribution 4.0 International License. Read Full License

Version of Record: A version of this preprint was published at Nature Communications on March 11th, 2022. See the published version at https://doi.org/10.1038/s41467-022-28886-5. 


\section{A Zirconium Metal-Organic Framework with SOC Topological Net for Catalytic Peptide Bond Hydrolysis}

Sujing Wang ${ }^{1,2,6 *}$, Hong Giang T. Ly ${ }^{3,4,6}$, Mohammad Wahiduzzaman ${ }^{5,6}$, lurii Dovgaliuk ${ }^{1}$, Antoine Tissot ${ }^{1}$, Guillaume Maurin ${ }^{5}$, Tatjana N. Parac-Vogt ${ }^{3 *}$ and Christian Serre ${ }^{1 *}$

${ }^{1}$ Institut des Matériaux Poreux de Paris, Ecole Normale Supérieure, ESPCI Paris, CNRS, PSL Université, Paris, France

${ }^{2}$ CAS Key Laboratory of Microscale Magnetic Resonance, Hefei National Laboratory for Physical Sciences at the Microscale, Suzhou Institute for Advanced Research, University of Science and Technology of China, Hefei, China

${ }^{3}$ Laboratory of Bioinorganic Chemistry, Department of Chemistry, KU Leuven, Leuven, Belgium.

${ }^{4}$ Department of Chemistry, College of Natural Sciences, Can Tho University, Can Tho, Vietnam.

${ }^{5}$ ICGM, Univ. Montpellier, CNRS, ENSCM, Montpellier, France.

${ }^{6}$ These authors contributed equally: Sujing Wang, Hong Giang T. Ly, Mohammad Wahiduzzaman

\section{*E-mail: sjwang4@ustc.edu.cn; tatjana.vogt@kuleuven.be; christian.serre@ens.fr}




\section{ABSTRACT}

The discovery of nanozymes for selective fragmentation of proteins would boost the emerging areas of modern proteomics, however, the development of efficient and reusable artificial catalysts for peptide bond hydrolysis is challenging. Here we report the detailed catalytic properties of a microporous zirconium carboxylate metal-organic framework, MIP-201, in promoting peptide bond hydrolysis in a simple dipeptide, as well as in horse-heart myoglobin (Mb) protein that consists of 153 amino acids. We demonstrate that MIP-201 features an excellent catalytic activity and selectivity, a good tolerance toward reaction conditions covering a wide range of different $\mathrm{pH}$ values, and importantly, an exceptional recycling ability associated with easy regeneration process. Taking into account the excellent catalytic performance of MIP-201 and its other advantages such as 6-connected $\mathrm{Zr}_{6}$ cluster active sites, the green, scalable and cost-effective synthesis, and an outstanding chemical and architectural stability, our finding suggests that MIP-201 may be a promising and practical alternative to the current commercially available catalysts for peptide bond hydrolysis.

\section{(3)}




\section{INTRODUCTION}

Peptide bond is a type of robust amide bond that connects amino acid residues in proteins, and thus it has essential importance in biological systems. The remarkable stability of peptide bond under physiological conditions (with an estimated half-life of $350-600$ years at $25{ }^{\circ} \mathrm{C}$ in neutral $\mathrm{pH}$ conditions) guarantees the intactness of the primary sequence of a protein, but results in a considerable challenge when there is a need to break the bond ${ }^{1}$.

Catalytic hydrolysis is an efficient way of breaking peptide bond, which results in the release of the carboxylic and amine functional groups. In biological systems this reaction is carried out by enzymes with extremely high reaction rates. However, outside biological systems, the hydrolysis of proteins is also an important procedure in areas such as protein structure analysis, protein engineering and protein-cleaving drug design ${ }^{2,3,4}$. Therefore, the high costs and the extreme sensitivity of enzymes to the reaction conditions motivated the development of artificial proteases ${ }^{2,3}$. The main goal in developing artificial proteases is to achieve adequate reactivity and specificity, as it is very challenging for artificial catalysts to match the exceptional catalytic power of natural enzymes.

Among existing alternatives, homogeneous catalysts based on Lewis acid metal salts often suffer from formation of gels at neutral and basic conditions, leading to loss of reactivity and difficulties in the separation of products and 
reactants ${ }^{5}$. In comparison, metal complexes prevent the formation of gels during catalysis, but have other shortcomings, such as limited reactivity window, toxicity and poor recyclability ${ }^{2,}{ }^{5}$. More recently metal-substituted polyoxometalate (POM) clusters were developed as homogeneous catalysts for peptide bond hydrolysis. In particular, zirconium(IV)-substituted POMs have been reported to achieve an encouraging combination of activity and selectivity in mildly acidic and neutral media6, 7, 8. But the structural dynamic of $\mathrm{Zr}$ substituted POMs under the reaction conditions and their high solubility make the catalyst recycling and product purification problematic. In order to circumvent these shortcomings, insoluble $\mathrm{Zr}_{6}$-0xo cluster-based metal-organic framework (MOF) were evaluated as the state-of-the-art heterogeneous catalyst for accelerating peptide bond hydrolysis ${ }^{5,9,10}$. The large-pore MOF-808 and NU-1000 (NU, Northwestern University) displayed much better reactivity and recyclability compared to POMs under neutral and mildly acidic conditions, but still suffer from limited stability under alkaline reaction conditions ${ }^{11,12}$ The highly defective UiO-66 (UiO, University of Oslo) and its functional derivatives, on the other hand, showed comparable catalytic performance with POMs but with improved stability under neutral and mildly alkaline conditions. Therefore, it still remains a great challenge to develop an efficient heterogeneous catalyst that is effective under various conditions and within a wide range of $\mathrm{pH}$ values, while preserving a good catalytic activity and specificity for the catalytic peptide bond hydrolysis. 
Herein, we present the first Zr-MOF with square-octahedron (soc) topological net constructed from $\mathrm{Zr}_{6}$-oxo cluster secondary building units (SBUs) and a tetracarboxylate linker $\left(3,3^{\prime}, 5,5^{\prime}\right.$-tetracarboxydiphenylmethane $\left.\left(\mathrm{H}_{4} \mathrm{mdip}\right)\right)$, denoted as MIP-201 (MIP, Materials of the Institute of Porous Materials from Paris), that addresses the challenge of developing highly efficient and robust heterogeneous catalyst for peptide bond hydrolysis. MIP-201 possesses catalytically active 6-connected $\mathrm{Zr}_{6}$-oxo cluster building units in its robust microporous network, which leads to excellent heterogeneous catalytic performance in accelerating peptide bond hydrolysis under a wide $\mathrm{pH}$ range of condition (acidic, neutral and basic), a good catalytic activity and selectivity, and a superior catalyst recycling ability. The combined advantage of its catalysis performance, along with its cost-efficient, green and scalable synthesis, and an excellent architectural and chemical stability, make MIP-201 one of the most promising artificial proteases discovered so far.

\section{RESULTS}

Synthesis and crystal structure of MIP-201. $\mathrm{Zr}_{6}-\mathrm{oxo}$ cluster is the dominant building unit in the fabrication of Zr-carboxylate MOFs. The connection numbers of $\mathrm{Zr}_{6}$-oxo clusters cover a wide range of $12,10,9,8,6,5$ and 4 in the reported examples, showing an extraordinary flexibility in connecting various linkers to generate a large library of different structures ${ }^{13,14,15,16}$. While the stabilities of Zr-MOFs constructed with low connection-number (5, and 4$)$ nodes are still under debate ${ }^{15,16,17,18}, 6$-connection is likely the limit to keep the material 
stability at an acceptable level toward certain applications ${ }^{19,20,21}$. In a sharp contrast to the large number of Zr-MOFs based on high connection-number

108 (above 8 till 12) building units, only six compounds are constructed from 6connected $\mathrm{Zr}_{6}-\mathrm{O}$ xo clusters, including PCN-22422, PCN-77723 (PCN, Porous

110 Coordination Network), UMCM-30924 (UMCM, University of Michigan 111 Crystalline Material), the interpenetrated Zr-BTB ${ }^{25}$, MOF- $808^{19}$ and BUT-108 112 (BUT, Beijing University of Technology) ${ }^{26}$.

113 In those structures, the 6-connected Zr6-oxo cluster shows two different 114 configuration modes, namely the hexagonal planar and trigonal prismatic. The 115 hexagonal planar configuration was only observed in PCN-224 while the other 116 five examples all share the trigonal prismatic one. The trigonal prismatic 117 configuration of the 6-connected $\mathrm{Zr}_{6}$-Oxo cluster is of a particular interest for the 118 design of new topological structure of $\mathrm{Zr}-\mathrm{MOFs}^{27}$, since it has been proven that 119 it could function similarly as the trimer building units of trivalent metal ions in 120 the MOF framework fabrication, such as the case of PCN-777 to be related to 121 the metal oxo-trimer-based MIL-100 and MIL-101 (MIL, Materials of Institut 122 Lavoisier $)^{23}$

123 In this regard, we have analyzed the possibility of replacing trivalent metal 124 trimer SBUs by 6-connected $\mathrm{Zr}_{6}$-Oxo clusters in several types of known MOF 125 structures, including MIL-88 ${ }^{28}$, soc-MOFs ${ }^{29}$ and series of PCN networks ${ }^{30}$. It 126 turned out that the soc topological net would be one of the most feasible targets 127 to achieve as detailed below: 1) all the connection sites on the trimer node are 
occupied by the carboxylate groups from the tetracarboxylate linker molecules, leading to the same configuration of the trigonal prismatic 6-connected $\mathrm{Zr6}$-oxo cluster; 2) the special shape of the tetracarboxylate linkers with appropriate steric hindrance and the separation between carboxylate groups could efficiently force the linkage and structure extending in the way of generating soc net; 3) reported soc-MOFs displayed excellent stability even with trivalent metal ions probably due to the hydrophobicity of the structural frameworks as well as the considerable steric hindrance around the SBU to weaken the attack from water molecules ${ }^{31,32}$. Regarding the selection of linker for this hypothesis, a tetracarboxylate ligand with an appropriate structural flexibility would be even beneficial if the larger size and elevated rigidity of $\mathrm{Zr}_{6}$-oxo cluster are taken into consideration in comparison with that of trivalent trimers. To that end, $\mathrm{H}_{4}$ mdip was finally selected as the most suitable tetracarboxylate linker for the synthesis of soc-Zr-MOF, not only due to its cost-effective and scalable synthesis, but also as its good structural flexibility to adapt various connection environments in the MOF construction is well documented ${ }^{33,} 34$.

Following the aforementioned guidance, a highly crystalline product (MIP-201) could be isolated from the solvothermal reaction of $\mathrm{ZrCl}_{4}$ and $\mathrm{H}_{4}$ mdip in pure acetic acid at $120^{\circ} \mathrm{C}$. Several attempts to obtain larger product particle suitable for single crystal X-ray diffraction data collection, including the combination of different $\mathrm{Zr}(\mathrm{IV})$ precursors with various solvent mixtures (formic acid, acetic anhydride, water, ethanol, etc.), did not lead to any increase in size of the 
product (Supplementary Fig. 1). In addition, the good tolerance of MIP-201

151 toward the synthesis conditions allows the development of green and scalable

152 routes for further practical applications. After optimization, an all-green reaction

153 of refluxing non-corrosive $\mathrm{Zr}\left(\mathrm{SO}_{4}\right)_{2} \cdot 4 \mathrm{H}_{2} \mathrm{O}$ and $\mathrm{H}_{4}$ mdip in the mixture of water

154 and acetic acid under ambient pressure was set for the scale-up synthesis of

155 MIP-201 product at the $10 \mathrm{~g}$ scale associated with a good yield ( $90 \%$ based on

156 the linker used).

a

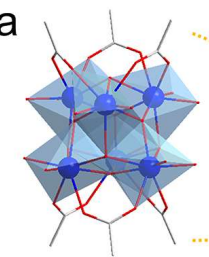

b

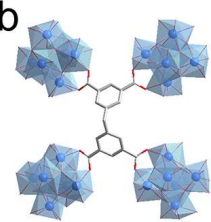

C

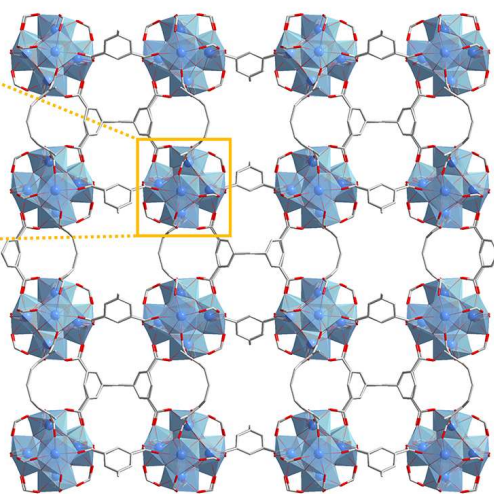

d

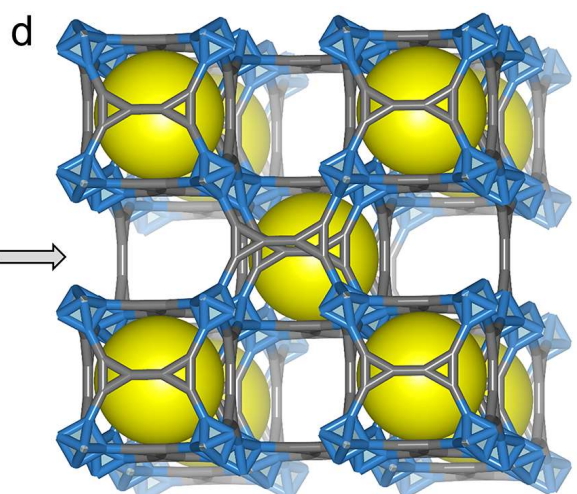

Fig.1 | Crystal structure of MIP-201. a, The 6-connected $\mathrm{Zr}_{6}$-oxo cluster building unit with the trigonal prismatic configuration. $\mathbf{b}$, One tetracarboxylate linker molecule connects the adjacent four $\mathrm{Zr}_{6}$-oxo clusters. c, The overall structure viewed along the c-axis. $\mathbf{d}$, The soc topological net of MIP-201 structure ( $Z r$ in blue, $C$ in gray, $\mathrm{O}$ in red, and the yellow ball stands for the cavity).

The crystal structure of MIP-201 was solved by a combined analysis of highresolution powder X-ray diffraction (PXRD) data and a computational topologyguided reverse engineering approach (Supplementary Figs. 2 and 3, Supplementary Table 1). MIP-201 with a formula of $\left[\mathrm{Zr}_{6}\left(\mu_{3}-\mathrm{O}\right)_{4}\left(\mu_{3-}\right.\right.$ $\mathrm{OH})_{4}(\text { acetate })_{0.24}(\mathrm{OH})_{5.76}\left(\mathrm{H}_{2} \mathrm{O}\right)_{5.76}$ (mdip) $)_{1.5}$, was found to crystalize in a cubic Im-3 space group (No. 204) with unit cell parameters of $a=24.5847(11) \AA$ and 
$V=14859.2(11) \AA^{3}$. It features a three-dimensional (3D) microporous framework composed of 6-connected $\mathrm{Zr}_{6}\left(\mu_{3}-\mathrm{O}\right)_{4}\left(\mu_{3}-\mathrm{OH}\right)_{4}$ oxo-cluster SBUs with mdip tetratopic linker molecules separating and spacing. As shown in Fig. 1a, each $\mathrm{Zr}_{6}$ oxo-cluster SBU comprises six carboxylate groups from six different linker molecules at the polar positions and six terminal acetate groups or -

$173 \mathrm{OH} / \mathrm{H}_{2} \mathrm{O}$ pairs locating around the equatorial plane. Taking account of the

174 molecular conformation character of mdip over the other rigid tetratopic linkers,

175 the considerable flexibility of the methylene group that connects two benzene 176 rings plays an important role in this case by forcing the mdip molecule to adapt 177 this connection mode (Fig. 1b). MIP-201 represents, to our knowledge, the first 178 example of this type of 6-connected Zr6 cluster SBU in Zr-MOFs built with 179 tetratopic linkers.

180 The four carboxylate groups of mdip linker are fully deprotonated to connect eight separated $\mathrm{Zr}(\mathrm{IV})$ ions binding four adjacent SBUs together (Fig. 1b). As a result of the high flexibility of the methylene group, mdip molecules are able to 183 adjust their length and width according to the corresponding connection requirements, and thus realize the interconnection of eight neighboring SBUs, giving rise to a distorted cubic pocket with a free diameter around $6 \AA$. However,

186 the windows size of the pocket is too small $(2.4 \AA)$ to be accessible for guest 187 molecules. There are however free voids generated between the neighboring 188 pockets showing an accessible dimension of around $10 \AA \times 12 \AA$, leading to a theoretical nitrogen-accessible surface area of $1040 \mathrm{~m}^{2} \mathrm{~g}^{-1}$ and a total free pore 
volume of $0.47 \mathrm{~cm}^{3} \mathrm{~g}^{-1}$ calculated from the crystal structure (Supplementary Fig. 4). However, residual $\mathrm{SO}_{4}{ }^{2-}$ groups are still trapped inside the pore of the MOF $(S / Z r=27.6 / 72.4$, atomic ratio), as evidenced by scanning electron microscopy with energy-dispersive X-ray spectroscopy (SEM-EDX) measurement, which results in an experimental Brunauer-Emmett-Teller (BET) area of $680 \mathrm{~m}^{2} \mathrm{~g}^{-1}$ and a total pore volume of $0.30 \mathrm{~cm}^{3} \mathrm{~g}^{-1}$ deduced from nitrogen porosimetry, slightly below the theoretical values. The similar observation of strongly trapped $\mathrm{Cl}^{-}$species in the MOF pore was found when $\mathrm{ZrCl}_{4}$ or $\mathrm{ZrOCl}_{2} \cdot 8 \mathrm{H}_{2} \mathrm{O}$ were used as the reactants for the synthesis of MIP-201, which has been recognized as a general issue for the fully activation of Zr-MOFs. The overall 3D structure of MIP-201 is fabricated through the cubic pockets packing to each other separated perfectly with the free void spaces (Fig. 1c), thus displays a typical network of cdj topology. It is worthy to note that MIP-201 is, to our knowledge, the first example of soc type network in Zr-MOFs (Fig. 1d).

Stability of MIP-201. In order to ensure the potential of MIP-201 in various applications, thermal and chemical stability tests under different conditions were carried out. Temperature-dependent PXRD and thermogravimetric analysis (TGA) data (Supplementary Figs. 5 and 6) indicate that MIP-201 maintains its crystal structure up to $375^{\circ} \mathrm{C}$, which is adequate for most of the applications related. Chemical resistance of MIP-201 has been checked under diverse conditions, including boiling water, fuming acid, super-acid, and basic conditions. As shown in Fig. 2a, the long-range order of the MIP-201 structure 
was maintained very well under all the tested conditions supported by their almost identical PXRD patterns. Long duration contacts with boiling water,

214 fuming acids, aqua regia, highly concentrated $\mathrm{H}_{3} \mathrm{PO}_{4}$ and basic conditions $215\left(\mathrm{pH}=10\right.$ buffer and $\mathrm{NH}_{4} \mathrm{OH}$ vapor) did not show notable degradation of the crystalline structure of MIP-201. Nitrogen sorption measurements at $77 \mathrm{~K}$ carried out on samples treated with some extremely harsh conditions supported

218 the good reservation of the MOF long-range order and porosity (Fig. 2b), despite of structural defects were generated in some cases such as $\mathrm{HCl}$ or aqua regia treatments. Therefore, MIP-201 displays excellent stability for applications, especially in a regard of bio-related applications, which generally requires a good tolerance toward the presence of phosphate species.
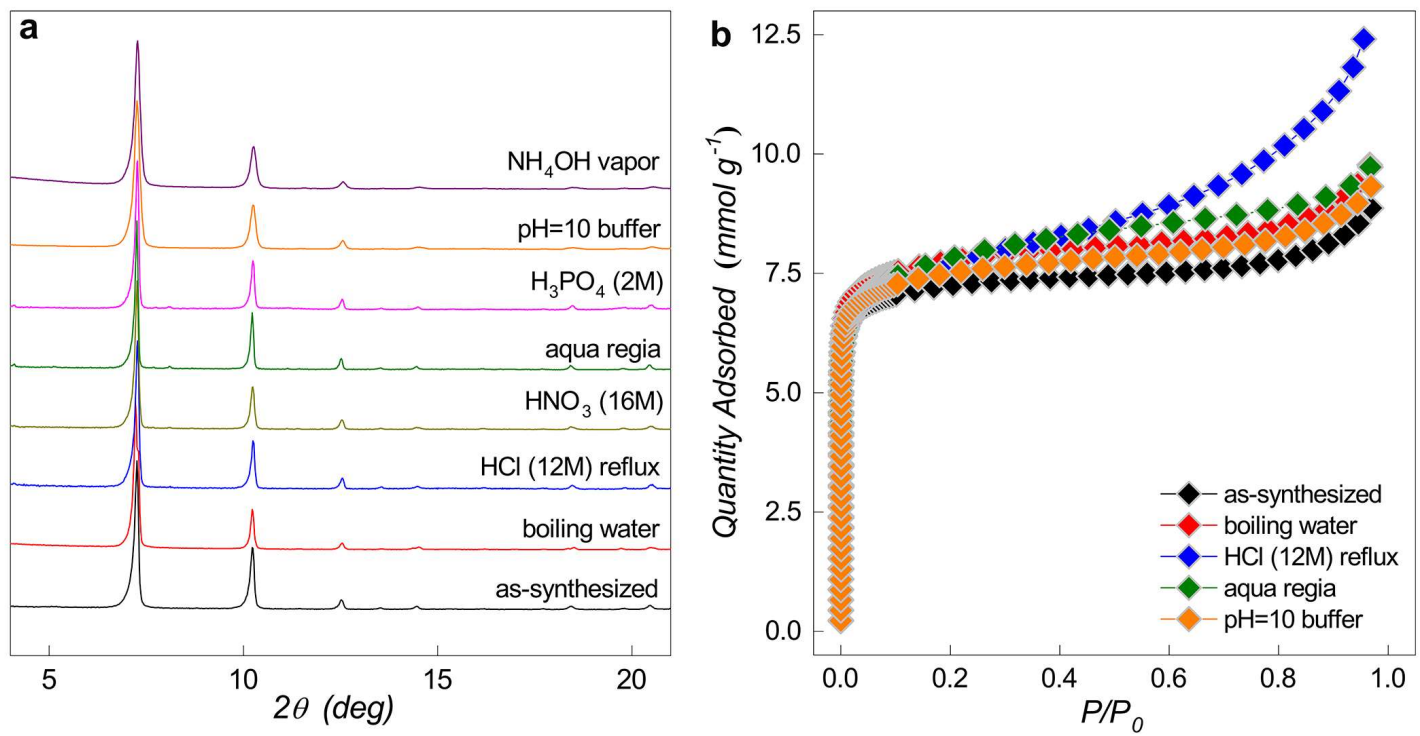

Fig. 2 | Chemical stability of MIP-201. a, PXRD patterns $\left(\lambda_{c u} \approx 1.5406 \AA\right)$ of MIP-201 samples treated under various chemical conditions. $\mathbf{b}$, Nitrogen adsorption isotherms of MIP201 samples after some typical chemical treatments collected at 77K (samples were refluxed in water before thermal activation at $120^{\circ} \mathrm{C}$ for each condition). 
The initial evaluation of the MIP-201 catalytic performance towards peptide bond hydrolysis was tested using a simple Gly-Gly dipeptide. A mixture of equimolar amounts $(2.0 \mu \mathrm{mol})$ of Gly-Gly and MIP-201 was incubated at $60{ }^{\circ} \mathrm{C}$ and pD 7.4 (Supplementary Fig. 7). and the progress of peptide bond hydrolysis was followed by ${ }^{1} \mathrm{H}$ NMR spectroscopy (Supplementary Fig. 8 and 9). To confirm that the catalytic activity was due to the MIP-201 material, and was not caused by $\operatorname{Zr}(\mathrm{IV})$ ions leached in solution, the MIP-201 powder was removed from the reaction mixture after 14 hours by centrifugation, and the homogeneous solution was allowed to react further. As it can be seen from Fig. 3a, no additional Gly-Gly hydrolysis was observed after removing MIP-201 from the reaction mixture, indicating that the peptide bond hydrolysis is exclusively associated with catalytically active sites in the solid MIP-201 material, and is not caused by $\mathrm{Zr}(\mathrm{IV})$ ions or Zr6-oxo clusters that might have leached into 242 solution.

243 The rate constant of Gly-Gly hydrolysis (kobsd) catalyzed by MIP-201 was 244 calculated to be $1.85 \times 10^{-5} \mathrm{~s}^{-1}$ (corresponding to $\mathrm{t}_{1} \frac{1}{2}=10.4 \mathrm{~h}$ ) at $60{ }^{\circ} \mathrm{C}$ and $\mathrm{pD}$ 7.4 (Supplementary Fig. 8), which represents an enhancement of nearly two orders of magnitude compared to the reactions catalyzed by $\operatorname{Zr}(\mathrm{IV})$-POMs 247 under the same experimental conditions ${ }^{6,7}$, and a 2500 times enhancement 248 compared to the un-catalyzed hydrolysis of Gly-Gly (kobsd=7.4 $\times 10^{-9} \mathrm{~s}^{-1}$, 249 corresponding to $t_{1 / 2}=3$ years). It is noteworthy that the rate of Gly-Gly 
hydrolysis by MIP-201 is comparable to that previously observed with MOF-808, when the larger surface area of MOF-808, which allows for more adsorption of peptide substrate, is taken into consideration (Supplementary Fig. 10). Furthermore, NU-1000, which has mesoporous cavities associated with a large BET area $\left(2200 \mathrm{~m}^{2} \mathrm{~g}^{-1}\right)$ but is constructed from 8-connected $\mathrm{Zr6} 6$-Oxo SBUs, showed a peptide bond hydrolysis rate which was more than one order of magnitude slower than that of MIP-201. This highlights the important role of 6connection of the $\mathrm{Zr}_{6}$-oxo node on the catalytic activity, and the fact that the active site density is critical for the efficiency of a MOF as artificial peptidase. Interestingly, the hydrolysis of amide bond in N-Methylacetamide by MIP-201 was not observed after 8 days of reaction (Supplementary Fig. 11), supporting the fact that the C-terminal carboxyl group plays an important role in acceleration of the reaction, possibly by enabling binding of the dipeptide to the catalytically active $\mathrm{Zr}(\mathrm{IV})$ sites.

The catalytic hydrolysis of peptide bond was further investigated as a function of $\mathrm{pD}$. The mixture of Gly-Gly and MIP-201 were incubated at $60^{\circ} \mathrm{C}$ in solutions with $\mathrm{pH}$ ranging from 3.9 to 9.9 and the corresponding results are presented in Fig. $3 b$. In the range from acidic to neutral $p D$, the rate enhancement was 268 observed along with the pD increase. Similar evolution was observed in other 269 catalytic systems, and was explained by the gradual reduction of Gly-Gly 270 protonation which facilitates the effective binding to the catalytically active metal 271 ion centers ${ }^{2}$. Remarkably, with further $\mathrm{pD}$ increase, a very distinct catalytic 

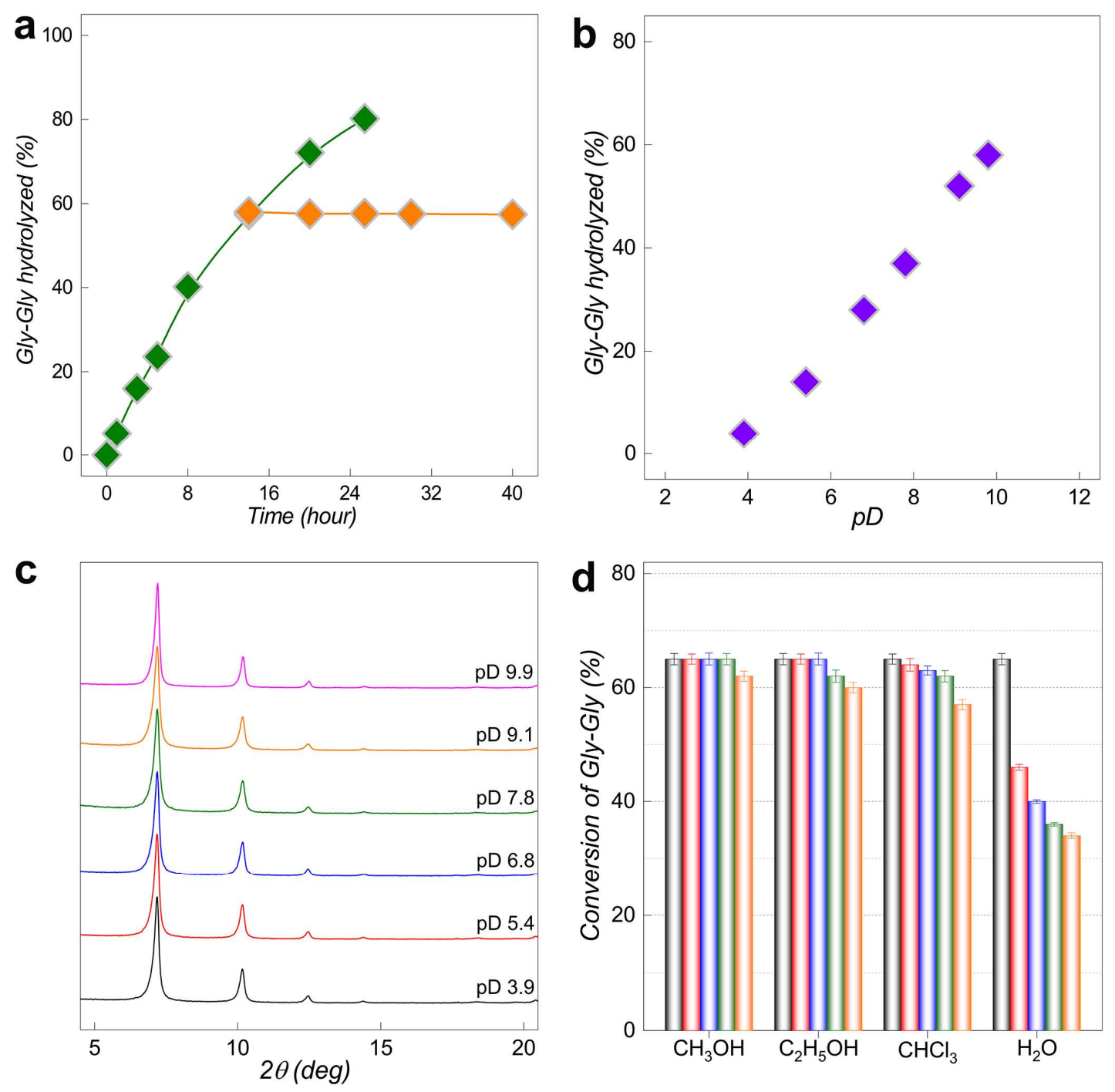

273 Fig. 3 | Catalytic performance of MIP-201 for hydrolysis of Gly-Gly. a. Hydrolysis of 2.0

$\mu \mathrm{mol}$ of Gly-Gly in the presence of $2.0 \mu \mathrm{mol}$ of MIP-201 before $(\diamond)$ and after $(\diamond)$ the removal of MIP-201 (pD 7.4 and $60^{\circ} \mathrm{C}$ ). b. Conversion of Gly-Gly after eight-hour hydrolysis of Gly-Gly (2.0 $\mu \mathrm{mol})$ catalyzed by MIP-201 $(2.0 \mu \mathrm{mol})$ at $60^{\circ} \mathrm{C}$ and different $\mathrm{pD}$ values. c. PXRD patterns of MIP-201 after eight-hour catalysis at $60^{\circ} \mathrm{C}$ in reaction solution of different $\mathrm{pD}$ values $\left(\lambda_{\mathrm{c} u} \approx\right.$ $1.5406 \AA$ ). d. Conversion of Gly-Gly after sixteen hours at $60^{\circ} \mathrm{C}$ in the presence of MIP-201 for

279 five reaction cycles. Different organic solvents were used to wash and exchange with water before a four-hour activation at $120^{\circ} \mathrm{C}$. 
steady increase, with a more than $50 \%$ rate enhancement observed at $\mathrm{pD}=9.9$ compared to physiological pD. On the contrary, decrease in reaction rates has been reported for many other catalysts that were used under alkaline conditions, and were mainly attributed to the instability of the catalysts. For instance, metal salts typically form inactive insoluble gels or solids under mildly basic solutions ${ }^{5}$, while POMs are largely unstable under alkaline conditions and show the best catalytic efficiency in slightly acidic situations ${ }^{6,7}$. Similarly, the PXRD patterns of both MOF-808 and NU-1000 revealed crystal structure degradation and reduced catalytic activities when the $\mathrm{pD}$ of the solution was raised above 8.0 (Supplementary Fig.12) ${ }^{5,9}$. On the contrary, the stability of MIP-201 within the full $\mathrm{pD}$ range of the reaction conditions was confirmed by PXRD (Fig. 3c) and IR spectroscopy (Supplementary Fig. 13) carried out on the MIP-201 samples collected after the completion of catalytic reaction. These characterizations indicated that the structure of MIP-201 remains intact with no evidence of degradation under all examined reaction conditions.

The recycling of MIP-201 as a heterogeneous peptidase was further investigated, revealing a remarkably high architectural stability after the direct regeneration by washing with water. Although a decrease of catalytic activity was observed for water-regenerated MIP-201, from $71 \%$ yield observed in the second run to $55 \%$ in the fourth run (Fig. 3 d), it could be rationalized by the microporous nature of the MIP-201 structure, in which a partial pore blocking may lead to a slight reduction of the number of accessible catalytically active 
sites. It is worth mentioning that comparable yields were observed in the fifth and fourth cycle, supporting the conclusion that the number of the accessible catalytic sites has approached an equilibrium while the overall architecture of the MIP-201 framework was maintained during the entire catalytic cycles. A similar observation was found when chloroform $\left(\mathrm{CHCl}_{3}\right)$ was used to regenerate catalyst after the reaction, while both methanol and ethanol were shown to be excellent solvents for regenerating MIP-201, in accordance to recyclability studies with other MOFs in general ${ }^{5}$. In contrast to MIP-201, the recycling of MOF-808 after Gly-Gly hydrolysis indicated a limited architectural stability of the MOF-808 framework, which impeded its regeneration by direct water washing and exchanging. This was attributed to the combined effect of considerable structural defects present in MOF-808 and the high surface tension of water ${ }^{5,11}$. This resulted in a drop of more than $90 \%$ in the second run of catalysis after washing with water, which was associated with a partial structural collapse of MOF-808 with the direct hydrolytic treatment.

Hydrolysis of Horse-heart myoglobin (Mb) catalyzed by MIP-201. As the high catalytic activity, good stability and recycling ability of MIP-201 were demonstrated in the hydrolysis of a model dipeptide, further evaluation of its

322 catalytic performance was carried out on Horse-heart myoglobin (Mb), a protein

323 containing 153 amino acids with approximate $\mathrm{Mw}$ of $16.95 \mathrm{kDa}$. Previous 324 studies have shown that water soluble $\mathrm{Zr}(\mathrm{IV})$ substituted $\mathrm{POMs}^{35}$ and insoluble $325 \mathrm{Hf}_{18}$ metal-oxo cluster ${ }^{36}$ were able to selectively hydrolyze $\mathrm{Mb}$ at peptide bonds 

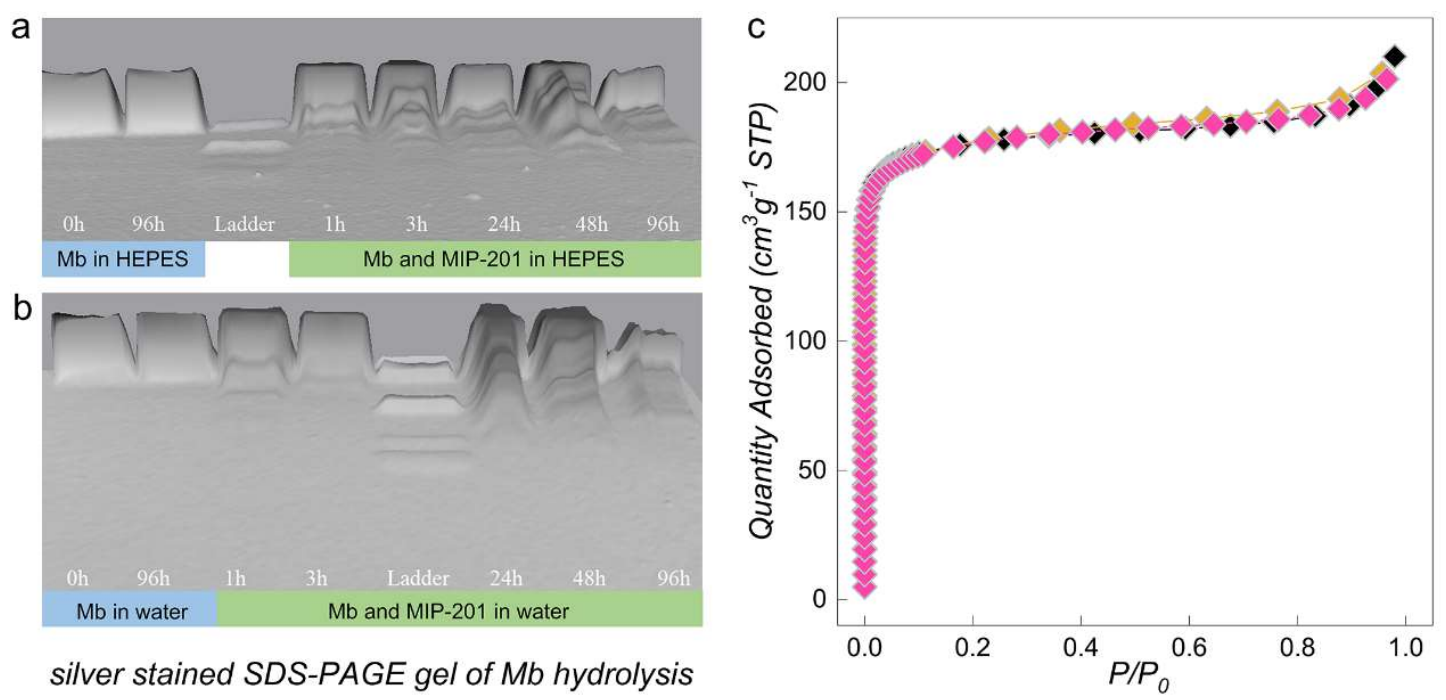

Fig.4 | Hydrolysis of Horse-heart myoglobin (Mb) catalyzed by MIP-201. a. Silver stained SDS-PAGE gel of Mb hydrolysis in the presence of MIP-201 in HEPES buffer (pH 7.4 and at $\left.60{ }^{\circ} \mathrm{C}\right)$. b. Silver stained SDS-PAGE gel of Mb hydrolysis in the presence of MIP-201 in water $\left(\mathrm{pH}\right.$ at 7.4 and at $\left.60^{\circ} \mathrm{C}\right)$. c. Nitrogen adsorption isotherms of MIP-201 samples before $(\bullet)$ and after the application in hydrolysis of Mb in HEPES buffer $(\diamond)$ and water $(\diamond)$.

artificial proteases. The reactions were carried out by mixing Mb with MIP-201 in HEPES buffer at $60{ }^{\circ} \mathrm{C}$ and analyzing reaction aliquots by SDS-PAGE at different time increments. The selective hydrolysis of $\mathrm{Mb}$ was evidenced by the appearance of new bands with lower molecular weights (Fig. 4a), and similar SDS-PAGE pattern was observed when pure water was used as medium (Fig. 4b). In the presence of MIP-201 the hydrolysis of Mb could be already observed after one hour of incubation, and the intensity of bands with lower molecular weight (MW) progressively increased with time. The MWs of the protein fragments formed by hydrolysis of Mb could be estimated using MW ladder in 
6.9 kDa, suggest that Mb was hydrolyzed at Asp20-Ile21, Asp44-lys45, Asp60-

Leu61 and Asp126-Asp127 peptide bonds, in accordance with the previously reported affinity of $\mathrm{Zr}(\mathrm{IV})$ based artificial proteases to preferentially cleave proteins next to Asp residues ${ }^{35}$. The hydrolysis of the same peptide bonds in $\mathrm{Mb}$ was also observed in the presence of $\mathrm{Zr}$-POMs, however, those reactions were much slower, with the observable hydrolysis occurring only after 48 hours at $\mathrm{pH}=5.0^{35}$. The control experiments showed that hydrolysis of $\mathrm{Mb}$ was not observed in the absence of MIP-201 after a four-day incubation at $60{ }^{\circ} \mathrm{C}$, confirming the catalytic role of MIP-201 in protein hydrolysis. The stability of MIP-201 after the reaction with Mb was evaluated by a combination of several techniques performed on samples collected from large-scale reactions. Nitrogen porosimetry (Fig. 4c), PXRD (Supplementary Fig. 14) and FTIR

356 (Supplementary Fig. 15) measurements confirmed that the structure of MIP201 remained intact after hydrolytic experiments with $\mathrm{Mb}$, further validating its potential as nanozyme for selective protein hydrolysis.

\section{Conclusions}

We demonstrate that the microporous MIP-201 reported in this work, which represents the first Zr-MOF with the soc type topological network, addresses several challenges related to the development of highly efficient and robust heterogeneous catalyst for peptide bond hydrolysis. An increase of more than three orders of magnitude was observed in the rate of the peptide bond 
hydrolysis in a model dipeptide, highlighting the importance of the 6connectivity of Zr6 oxo-cluster sites in the MIP-201 framework for catalytic efficiency. Compared to few other MOFs, the most striking advantage of MIP201 in catalyzing peptide bond hydrolysis is the possibility to use catalyst under wide range of reaction conditions, without compromising its stability. MIP-201 was shown to selectively hydrolyze myoglobin which has 153 amino acids in its sequence, selectively cleaving the protein at only four peptide bonds. The excellent chemical and architectural stability of MIP-201 confirmed in the experiments with the protein, in addition to its high catalytic activity and recycling ability, further highlights its potential as an artificial protease. Furthermore, the green, scalable and cost-effective synthesis of MIP-201 with a good product yield makes it a promising and practical alternative for selective hydrolysis of proteins in proteomics and biotechnology applications.

\section{Methods}

Synthesis of MIP-201. $\mathrm{Zr}\left(\mathrm{SO}_{4}\right)_{2} \cdot 4 \mathrm{H}_{2} \mathrm{O}(10.8 \mathrm{~g})$ and $\mathrm{H}_{4}$ mdip $(5.1 \mathrm{~g})$ were transferred to a round bottom flask $(1 \mathrm{~L})$, followed by the addition of water $(300 \mathrm{~mL})$ and acetic acid $(100 \mathrm{~mL})$ under stirring at room temperature. The reaction was refluxed at $120^{\circ} \mathrm{C}$ for 12 hours and cooled to R.T. The resulted solid product was collected by filtration, washed with ethanol and air dry. Crude product with a light gold color $(12.5 \mathrm{~g}, 90 \%$ yield based on $\mathrm{H}_{4}$ mdip) was obtained. 
and $60{ }^{\circ} \mathrm{C}$ by applying a general procedure. $\mathrm{D}_{2} \mathrm{O}(950 \mu \mathrm{L})$ was added to a solid sample of MIP-201 (2.8 mg, $2.0 \mu \mathrm{mol})$ in a glass vial followed by stirring for 30 minutes at room temperature. Peptide (50 $\mu \mathrm{L}$ of $40 \mathrm{mM}$ stock solution, $2.0 \mu \mathrm{mol}$ ) was added to the suspension and the $\mathrm{pH}$ of reaction mixture was adjusted to $\mathrm{pD} 7.4$ by using $\mathrm{NaOD}$. The samples were incubated at $60{ }^{\circ} \mathrm{C}$. After different time increments the reaction mixture was centrifuged at $15000 \mathrm{rpm}$ for $20 \mathrm{~min}$ to remove the MOF. The ${ }^{1} \mathrm{H}$ NMR spectra of the resulting solutions were recorded using TMSP-d4 as internal reference. The rate constants were obtained by fitting peptide concentrations at different time increments to a first-order decay function.

Protein hydrolysis studies. $\mathrm{Mb}(0.02 \mathrm{mM})$ was mixed with $2.8 \mathrm{mg}$ of MIP-201 in HEPES buffer at $\mathrm{pH} 7.4$ or in pure water at $\mathrm{pH} 7.4$. Samples were stirred at $60^{\circ} \mathrm{C}$ and aliquots for SDS-PAGE analysis were taken at different time increments. Stacking gel was $4 \%(\mathrm{w} / \mathrm{v})$ polyacrylamide in $0.5 \mathrm{M}$ Tris- $\mathrm{HCl}$ buffer at $\mathrm{pH} 6.8$ and resolving gel consisted of $18 \%(\mathrm{w} / \mathrm{v})$ polyacrylamide in $1.5 \mathrm{M}$ Tris- $\mathrm{HCl}$ buffer at $\mathrm{pH}$ 8.8. Sample buffer $(5 \mu \mathrm{L})$ was added to $15 \mu \mathrm{L}$ of the reaction mixture and after heating at $100^{\circ} \mathrm{C}$ for $5 \mathrm{~min}$, used in SDS-PAGE was Page Ruler unstained low range protein. An OmniPAGE electrophoretic cell was combined with an EV243 power supply and the experiments were performed at $200 \mathrm{~V}$ for $1.5 \mathrm{~h}$.

Catalyst recycling experiments. Recyclability of MIP-201 was tested by repeating Gly-Gly hydrolysis five times starting from one batch of catalyst in a glass vial. After each run, the reaction mixture was centrifuged and the solution was used to analyze 

by ${ }^{1} \mathrm{H}$ NMR. Water was added to the solid MOF and stirred for one hour before removing it. This process was repeated for four times. The MOF material was subsequently stirred in an organic solvent (chloroform, ethanol, methanol...) for one day to exchange water. The procedure was repeated twice and the recycled MIP-201 was air dried and activated at $120{ }^{\circ} \mathrm{C}$ for four hours before being used for the next catalytic run.

\section{Data availability}

All data involved in this work are included in this article and the corresponding supplementary information. They are available from the corresponding authors upon reasonable request. The crystal structure data have been deposited at CCDC under the deposition numbers CCDC: 2076200 and 2076201. These data can be obtained free of charge from the CCDC database via www.ccdc.cam.ac.uk.

\section{References}

1. Radzicka, A. \& Wolfenden, R. Rates of Uncatalyzed Peptide Bond Hydrolysis in Neutral

2. Wezynfeld, N.E., Frączyk, T. \& Bal, W. Metal assisted peptide bond hydrolysis: Chemistry, biotechnology and toxicological implications. Coordination Chemistry Reviews 327-328,

3. Kathryn, B.G. \& Miki, K. Major Advances in the Hydrolysis of Peptides and Proteins by Metal lons and Complexes. Current Organic Chemistry 10, 1035-1049 (2006). 
5. Ly, H.G.T. et al. Superactivity of MOF-808 toward Peptide Bond Hydrolysis. Journal of the American Chemical Society 140, 6325-6335 (2018).

6. Absillis, G. \& Parac-Vogt, T.N. Peptide Bond Hydrolysis Catalyzed by the Wells-Dawson

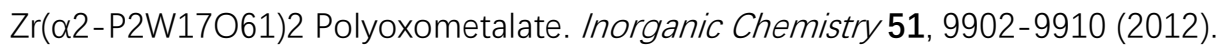

7. Ly, H.G.T., Mihaylov, T., Absillis, G., Pierloot, K. \& Parac-Vogt, T.N. Reactivity of Dimeric Tetrazirconium(IV) Wells-Dawson Polyoxometalate toward Dipeptide Hydrolysis Studied by a Combined Experimental and Density Functional Theory Approach. Inorganic Chemistry 54, 11477-11492 (2015).

8. Ly, H.G.T. et al. Chemical Mimics of Aspartate-Directed Proteases: Predictive and Strictly Specific Hydrolysis of a Globular Protein at Asp - X Sequence Promoted by Polyoxometalate Complexes Rationalized by a Combined Experimental and Theoretical Approach. Chemistry - A European Journa/ 25, 14370-14381 (2019).

9. Loosen, A. et al. Interplay between structural parameters and reactivity of Zr6-based MOFs as artificial proteases. Chemical Science 11, 6662-6669 (2020).

10. Ly, H.G.T., Fu, G., de Azambuja, F., De Vos, D. \& Parac-Vogt, T.N. Nanozymatic Activity of UiO-66 Metal-Organic Frameworks: Tuning the Nanopore Environment Enhances Hydrolytic Activity toward Peptide Bonds. ACS Applied Nano Materials 3, 8931-8938 (2020).

11. Zhang, $X$. et al. A historical overview of the activation and porosity of metal-organic frameworks. Chemical Society Reviews 49, 7406-7427 (2020).

12. Wang, S. et al. A robust large-pore zirconium carboxylate metal-organic framework for energy-efficient water-sorption-driven refrigeration. Nature Energy 3, 985-993 (2018).

13. Chen, Y. et al. Zirconium-Based Metal-Organic Framework with 9-Connected Nodes for Ammonia Capture. ACS Applied Nano Materials 2, 6098-6102 (2019).

14. Bai, Y. et al. Zr-based metal-organic frameworks: design, synthesis, structure, and applications. Chem Soc Rev 45, 2327-2367 (2016).

15. Wang, H. et al. Topologically guided tuning of $\mathrm{Zr}$-MOF pore structures for highly selective separation of C6 alkane isomers. Nature Communications 9, 1745 (2018).

16. Chen, Y. et al. Structural Diversity of Zirconium Metal-Organic Frameworks and Effect on Adsorption of Toxic Chemicals. Journal of the American Chemical Society 142, 21428 21438 (2020). 
17. Zhang, Y. et al. A Flexible Interpenetrated Zirconium-Based Metal-Organic Framework with High Affinity toward Ammonia. ChemSusChem 13, 1710-1714 (2020).

18. Zhang, Y. et al. A Flexible Metal-Organic Framework with 4-Connected Zr6 Nodes. Journal of the American Chemical Society 140, 11179-11183 (2018).

19. Furukawa, H. et al. Water adsorption in porous metal-organic frameworks and related materials. J Am Chem Soc 136, 4369-4381 (2014).

20. Jiang, J. et al. Superacidity in Sulfated Metal-Organic Framework-808. Journal of the American Chemical Society 136, 12844-12847 (2014).

21. Feng, M., Zhang, P., Zhou, H.-C. \& Sharma, V.K. Water-stable metal-organic frameworks for aqueous removal of heavy metals and radionuclides: A review. Chemosphere 209, 783-800 (2018).

22. Feng, D. et al. Construction of Ultrastable Porphyrin Zr Metal-Organic Frameworks through Linker Elimination. Journal of the American Chemical Society 135, 17105-17110 (2013).

23. Feng, D. et al. A Highly Stable Zeotype Mesoporous Zirconium Metal-Organic Framework with Ultralarge Pores. Angewandte Chemie International Edition 54, 149-154 (2015).

24. Ma, J., Wong-Foy, A.G. \& Matzger, A.J. The Role of Modulators in Controlling Layer Spacings in a Tritopic Linker Based Zirconium 2D Microporous Coordination Polymer. Inorganic Chemistry 54, 4591-4593 (2015).

25. Wang, R. et al. Porous Zirconium Metal-Organic Framework Constructed from 2D $\rightarrow$ 3D Interpenetration Based on a 3,6-Connected kgd Net. Inorganic Chemistry 53, 7086-7088 (2014).

26. Kong, X.-J. et al. Constructing new metal-organic frameworks with complicated ligands from "One-Pot" in situ reactions. Chemical Science 10, 3949-3955 (2019).

27. Schoedel, A. \& Zaworotko, M.J. [M3( $33-\mathrm{O})(\mathrm{O} 2 \mathrm{CR}) 6]$ and related trigonal prisms: versatile molecular building blocks for crystal engineering of metal-organic material platforms. Chemical Science 5, 1269-1282 (2014).

28. Férey, G. Structural flexibility in crystallized matter: from history to applications. Dalton Trans. 45, 4073-4089 (2016).

29. Alezi, D. et al. MOF Crystal Chemistry Paving the Way to Gas Storage Needs: AluminumBased soc-MOF for $\mathrm{CH} 4, \mathrm{O} 2$, and $\mathrm{CO} 2$ Storage. Journal of the American Chemical Society 137, 13308-13318 (2015). 
523

524

525

526

527

528

529

530

531

532

533

534

535

536

537

538

539

540

541

542

543

544

545

546

547

548

549

550

551

552

553

554

555

556

557

30. Feng, D. et al. Kinetically tuned dimensional augmentation as a versatile synthetic route towards robust metal-organic frameworks. Nature Communications 5, 5723 (2014).

31. Belmabkhout, Y. et al. Metal-organic frameworks to satisfy gas upgrading demands: finetuning the soc-MOF platform for the operative removal of H2S. Journal of Materials Chemistry A 5, 3293-3303 (2017).

32. Verma, G. et al. A robust soc-MOF platform exhibiting high gravimetric uptake and volumetric deliverable capacity for on-board methane storage. Nano Research 14, 512517 (2021).

33. He, Y., Li, B., O'Keeffe, M. \& Chen, B. Multifunctional metal-organic frameworks constructed from meta-benzenedicarboxylate units. Chemical Society Reviews 43, 56185656 (2014).

34. Wang, S. et al. A phase transformable ultrastable titanium-carboxylate framework for photoconduction. Nat Commun 9, 1660 (2018).

35. Ly, H.G.T., Absillis, G., Janssens, R., Proost, P. \& Parac-Vogt, T.N. Highly Amino Acid Selective Hydrolysis of Myoglobin at Aspartate Residues as Promoted by Zirconium(IV)Substituted Polyoxometalates. Angewandte Chemie International Edition 54, 7391-7394 (2015).

36. Moons, J. et al. Discrete Hf18 Metal-oxo Cluster as a Heterogeneous Nanozyme for SiteSpecific Proteolysis. Angewandte Chemie International Edition 59, 9094-9101 (2020).

\section{Acknowledgements}

The authors from France acknowledge the ANR Project MeaCoPA (ANR-17-CE290003) and the European Community Seventh Program (FP7/2007-2013) for funding the research under Grant Agreement 608490 (Project M4CO2). S.W. acknowledges support from the National Natural Science Foundation of China (22071234) and the Fundamental Research Funds for the Central Universities (WK2480000007).

\section{Author contributions}

Conceptualization, S.W., HGT.L., TN.P. and C.S.; Investigation, S.W., HGT.L., M.W., 
558 I.D., A.T., G.M., TN.P., and C.S.; Writing - Original Draft, S.W.; Writing - Review \&

559 Editing, S.W., HGT.L., M.W., G.M., TN.P., and C.S. Supervision, TN.P. and C.S.

560 Competing interests

561 The authors declare no competing interests. 


\section{Supplementary Files}

This is a list of supplementary files associated with this preprint. Click to download.

- 2076200.cif

- 2076201.cif

- Supplementarylnformation.pdf 